\title{
Fluorescence resonance energy transfer (FRET) in chemistry and biology: Non-Förster distance dependence of the FRET rate
}

\author{
SANGEETA SAINI, ${ }^{1}$ HARJINDER SINGH ${ }^{2}$ and BIMAN BAGCHI ${ }^{1} *$ \\ ${ }^{1}$ Solid State and Structural Chemistry Unit, Indian Institute of Science, Bangalore 560012 \\ ${ }^{2}$ Permanent address: Department of Chemistry, Panjab University, Chandigarh 160014 \\ e-mail: bbagchi@sscu.iisc.ernet.in
}

\begin{abstract}
Fluorescence resonance energy transfer (FRET) is a popular tool to study equilibrium and dynamical properties of polymers and biopolymers in condensed phases and is now widely used in conjunction with single molecule spectroscopy. In the data analysis, one usually employs the Förster expression which predicts $\left(1 / R^{6}\right)$ distance dependence of the energy transfer rate. However, critical analysis shows that this expression can be of rather limited validity in many cases. We demonstrate this by explicitly considering a donor-acceptor system, polyfluorene $\left(\mathrm{PF}_{6}\right)$-tetraphenylporphyrin $(\mathrm{TPP})$, where the size of both donor and acceptor is comparable to the distance separating them. In such cases, one may expect much weaker distance (as $1 / R^{2}$ or even weaker) dependence. We have also considered the case of energy transfer from a dye to a nanoparticle. Here we find $1 / R^{4}$ distance dependence at large separations, completely different from Förster. We also discuss recent application of FRET to study polymer conformational dynamics.
\end{abstract}

Keywords. Fluorescence resonance energy transfer (FRET); non-Förster regime; single molecule spectroscopy; donor-acceptor system.

\section{Introduction}

Resonance energy transfer (RET) is a widely prevalent photophysical process through which an electronically excited 'donor' molecule transfers its excitation energy to an 'acceptor' molecule (as depicted in figure 1) such that the excited state lifetime of the donor decreases. ${ }^{1,2}$ If the donor happens to be a fluorescent molecule RET is referred to as fluorescence resonance energy transfer, FRET. The acceptor however may or may not be fluorescent.

Solving the enigma surrounding fluorescence quenching experiments revealed the phenomenon of FRET and led J Perrin ${ }^{3}$ to propose dipole-dipole interactions as the mechanism via which molecules can interact without collisions at distances greater than their molecular diameters. Some 20 years later, Förster ${ }^{1}$ built upon Perrin's idea to put forward an elegant theory which provided a quantitative explanation for the non-radiative energy transfer in terms of his famous expression given by

$$
k_{F}=k_{\mathrm{rad}}\left(R_{F} / R\right)^{6},
$$

Dedicated to Prof J Gopalakrishnan on his 62nd birthday *For correspondence where $k_{\text {rad }}$ is the radiative rate (typically less than $10^{9} \mathrm{~s}^{-1}$ ) and $R_{F}$ is the well-known Förster radius given by the spectral overlap between the fluorescence spectrum of the donor and the absorption spectrum of the acceptor. Since then the technique of FRET has come a long way finding applications in most of the disciplines, which by itself signifies the importance of Förster's formulation and usefulness of this technique.

Undoubtedly, understanding any phenomenon on a molecular scale has always been one of the major goals of all physical, chemical and biological quests. However, at present when there is great interest in characterizing nano-materials, its achievement has gained immense priority as never before. To understand a phenomenon on a molecular scale requires information about the spatial relationships between the molecules, and this is where FRET's performance is the best, i.e. to quantitatively measure distances between molecules in the range of 10-100 $\AA$, thereby providing us with invaluable information about structures and dynamics of macromolecules.

In this paper, we shall address several aspects of FRET. The emphasis of this paper is on the distancedependence of the fluorescence energy transfer. Recent studies have shown that the usually accepted $R^{-6}$ distance dependence can be easily violated when the 
sizes of donor and/or acceptor are smaller than or comparable to the distance separating them. In $\$ 2$, we present a critical analysis of the assumptions which lead to the Förster distance dependence. Subsequently $\S \S 2$ and 3 , we briefly discuss the applications of FRET in several areas as, for example, in the study of the conformational dynamics of polymers and biopolymers. We also discuss how and why Förster distance dependence is violated in case of conjugated polymers. In $\S 4$, we present an analysis of the energy transfer from a dye to a nano-metal particle. Here also we find reasons for marked non-Förster distance-dependence. We conclude the paper with a brief discussion of the significance of the results.

\section{Principles underlying FRET}

Resonance energy transfer is a non-radiative quantum mechanical process and requires fluorescence emission spectrum of the donor molecule (D) to overlap with the emission spectrum of the acceptor (A), and the two to be within the minimal spatial range for the donor to transfer its excitation energy to the acceptor. The Förster theory is based on the

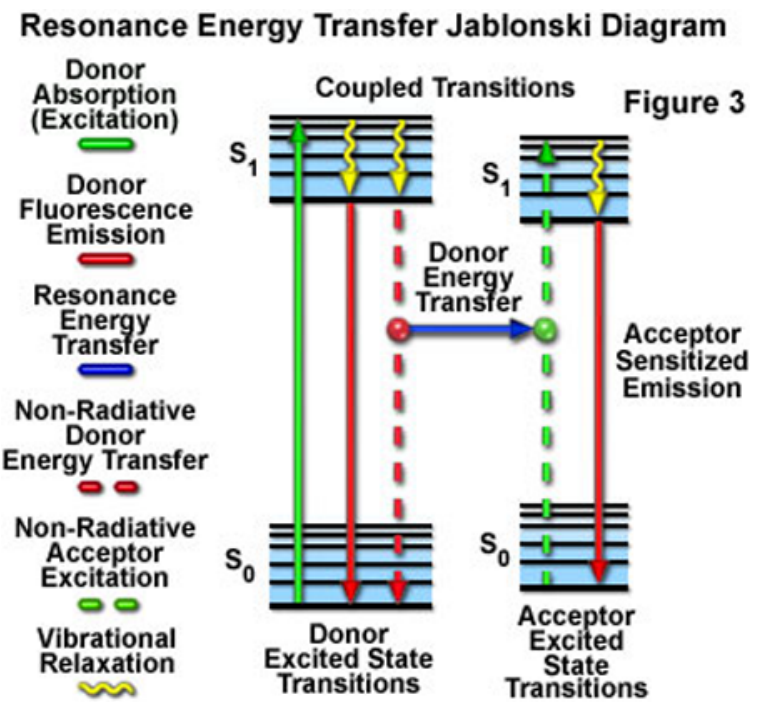

Figure 1. Jablonski diagram illustrating coupled transitions between donor emission and acceptor absorbance in fluorescence resonance energy transfer. Absorption and emission transitions are represented by straight vertical arrows (green and red respectively), while vibrational relaxation is indicated by wavy yellow arrows. The coupled transitions are drawn with dashed lines. The phenomenon of FRET is illustrated by a blue arrow (source: www.olympusmicro.com/primer/techniques/fluorescence/fret/fretintro.html). equilibrium Fermi-golden rule approach, where the transfer of excitation energy is regarded to be the transition between the electronic states $\phi_{e}^{D} \phi_{g}^{A}$ and $\phi_{g}^{D} \phi_{e}^{A}$ (where ' $g$ ' and ' $e$ ' stand for ground and excited state respectively) promoted via a coulombic interaction, a long-range dipole-dipole intermolecular coupling between D and A. The key assumptions of Förster formulation are: (a) A dipole-dipole approximation can be employed for electronic coupling between $\mathrm{D}$ and $\mathrm{A}$; (b) vibrational relaxation after electronic excitation of donor takes place on a much faster time-scale as compared to RET; (c) coupling of molecules to the surroundings is much stronger than coupling between D and A, ensuring that FRET is an irreversible and incoherent process. ${ }^{4}$ With these assumptions in mind, the Fermi-golden rule approach is followed to obtain the expression for energy transfer as derived below.

Consider the D-A system with D in an excited state, having $M$ vibronic energy levels which we consider to be associated with the excited state and A in the ground state with $N$ vibronic energy levels assumed to be associated with the ground state. The excitation energy transfer in DA complex is considered to be the transfer between the excited state wavefunction of donor, $\psi_{e}^{D}\left(r_{D} ; R_{D}\right)=\phi_{e}^{D}\left(r_{D} ; R_{D}\right) \chi_{D e}$, having energy $E_{\mathrm{DeM}}$ and the ground state of an acceptor $\psi_{g}^{A}\left(r_{A} ; R_{A}\right)=\phi_{g}^{A}\left(r_{A} ; R_{A}\right) \chi_{A g}$ with energy $E_{\mathrm{AgN}}$. According to Fermi-golden rule the transition rate is given by:

$$
\begin{aligned}
& k_{F}=\frac{2 \pi}{\hbar} \sum_{M_{D}} \sum_{N_{A}} f\left(E_{D e M_{D}}\right) f\left(E_{A g N_{A}}\right) \\
& \left|\left\langle\psi_{e M_{D}}^{D}, \psi_{g N_{A}}^{A}\left|V_{D A}\right| \psi_{e M_{A}}^{A}, \psi_{g N_{D}}^{D}\right\rangle\right|^{2} \\
& \times \delta\left(E_{D e M_{D}}+E_{A g N_{A}}-E_{A e M_{A}}-E_{D g N_{D}}\right),
\end{aligned}
$$

where $f\left(E_{\mathrm{DeM}}\right)$ and $f\left(E_{\mathrm{AgN}}\right)$ represent thermal distribution for initial vibrational states of the donor and acceptor, and $V_{D A}$ accounts for the coulomb interaction between donor and acceptor molecules. By assuming that coupling matrix elements do not depend on the nuclear coordinates (Condon approximation) we can write,

$$
\begin{aligned}
& \left|\left\langle\psi_{e M_{D}}^{D}, \psi_{g N_{A}}^{A}\left|V_{D A}\right| \psi_{e M_{A}}^{A}, \psi_{g N_{D}}^{D}\right\rangle\right| \\
& =J_{D A}\left\langle\chi_{D e M_{D}} \mid \chi_{D g N_{D}}\right\rangle\left\langle\chi_{A g N_{A}} \mid \chi_{A e M_{A}}\right\rangle,
\end{aligned}
$$


where $J_{D A}$ represents the elements of the electronic matrix which for dipole-dipole interaction is

$$
J_{D A}=k\left\{\left|\mathbf{d}_{e g}^{D} \| \mathbf{d}_{e g}^{A}\right| / R^{3} \mid\right\},
$$

$R$ is separation between donor and acceptor molecule, $\kappa$ is a dimensionless geometric factor and $d$ is transition dipole-moment vector. This rate expression can be expressed in terms of acceptor absorption and donor emission spectrum. To incorporate this, the delta function can be written as,

$$
\begin{aligned}
& \delta\left(E_{D e M_{D}}+E_{A g N_{A}}-E_{A e M_{A}}-E_{D g N_{D}}\right) \\
& =\int_{-\infty}^{+\infty} \delta\left(E_{D e M_{D}}-E_{D g N_{D}}-E\right) \times \delta\left(E+E_{A g N_{A}}-E_{A e M_{A}}\right) d E .
\end{aligned}
$$

Here the first delta function represents the donor emission while the second represents the acceptor absorption.

The frequency dependent absorption coefficient of the acceptor can be defined as,

$$
\begin{aligned}
a_{A}(\omega)= & \frac{4 \pi^{2} \omega \eta}{3 c} \\
& \left|\mathbf{d}_{e g}^{D}\right|^{2} \sum_{M_{A}, N_{A}} f\left(E_{A g N_{A}}\right)\left|\left\langle\chi_{A g N_{A}} \mid \chi_{A e M_{A}}\right\rangle\right|^{2} \\
& \times \delta\left(E_{A g N_{A}}-E_{A e M_{A}}+\hbar \omega\right) .
\end{aligned}
$$

Similarly, donor emission spectrum can be written as,

$$
\begin{aligned}
f_{D}(\omega)= & \frac{4 \omega^{3} \eta^{3}}{3 \hbar c^{3}}\left|d_{e g}^{D}\right|^{2} \\
& \sum_{M_{D}, N_{D}} f\left(E_{D e M_{D}}\right)\left|\left\langle\chi_{D e M_{D}} \mid \chi_{D g N_{D}}\right\rangle\right|^{2} \\
& \times \delta\left(E_{D e M_{D}}-E_{D g N_{D}}-\hbar \omega\right) .
\end{aligned}
$$

Substituting for these expressions in (2) and using (3) and (4) we get,

$$
k_{F}=\frac{9 \kappa^{2} c^{4}}{8 \pi \eta^{4}} \frac{1}{R^{6}} \int_{0}^{\infty} \frac{a_{A}(\omega) f_{D}(\omega) \mathrm{d} \omega}{\omega^{4}}
$$

where $\eta$ is the refractive index of the medium which affects all the electric interactions in condensed media and $c$ is the velocity of light.
From (6) we can see that the rate of FRET decreases as the sixth power of the distance between donor and acceptor which accounts for the technique's sensitivity in the range, with $100 \AA$ as the upper limit and $10 \AA$ as the lower limit, as below $10 \AA$ other modes of energy transfer are also possible.

$\kappa^{2}$, orientation factor is given by,

$$
\kappa^{2}=\left(2 \cos \theta_{D} \cos \theta_{A}-\sin \theta_{D} \sin \theta_{A} \cos \phi\right)^{2},
$$

where $\theta_{D}$ and $\theta_{A}$ are the angles which the dipoles of $\mathrm{D}$ and $\mathrm{A}$ form with the axis joining $\mathrm{A}$ and $\mathrm{D}$, with $\varphi$ being an angle between the corresponding planes as shown in figure 2 . Since orientation factor depends upon the relative orientations of the donor emission dipole and the acceptor absorption dipole, its value can range from 0 to 4 . However, determining the exact value of $\kappa^{2}$ is often difficult, and in most of the cases the dynamically averaged value of $2 / 3$ works well though its use is valid only under the assumption that both $\mathrm{D}$ and $\mathrm{A}$ are free to undergo unrestricted isotropic motion, which certainly is not entirely correct especially for macromolecules. Therefore, use of the $2 / 3$ value has always been controversial. This uncertainty can be minimized by fluorescence anisotropy measurements. ${ }^{5}$

The spectral overlap term in rate expression contains a lot of complicated information about the nuclear overlap factors separated from the electronic coupling term. The beauty of Förster formulation lies in the fact that this complicated information can be obtained from relatively simple experimental data.

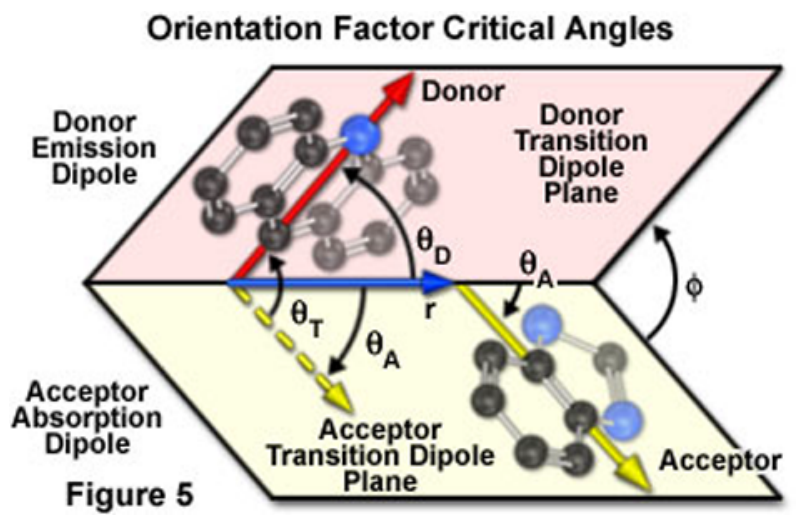

Figure 2. Illustration of dependence of the orientation factor $\left(\kappa^{2}\right)$ on the relative orientations of the donor emission dipole and the acceptor absorption dipole (source: www.olympusmicro.com/primer/techniques/fluorescence/ fret/fretintro.html). 
Equation (6) can also be written as

$$
k_{F}=\left(1 / \tau_{D}\right)\left(R_{F} / R\right)^{6}
$$

where $R_{F}$ is the Förster critical distance defined to be the distance at which the efficiency of EET from a donor to an acceptor becomes $50 \%$ and $\tau_{D}$ is donor lifetime in the absence of an acceptor and is the reciprocal of $k_{\text {rad }}$.

Experimentally, distance $R$ can be determined by measuring the efficiency of energy transfer $\left(E_{T}\right)$.

$$
R=R_{F} /\left(\left(1 / E_{T}\right)-1\right)^{6}
$$

where $E_{T}$ is evaluated as

$$
E_{T}=1-\left(\tau_{D A} / \tau_{D}\right)
$$

Here $\tau_{D A}$ is donor fluorescence lifetime in the presence of an acceptor.

\section{FRET in biopolymers: Applications to life sciences}

FRET has been extensively exploited in biological research and biotechnological applications for the past few decades.

The value of $R_{F}$ which in fact is the maximum separation distance between the donor and the acceptor molecules over which RET can still occur, normally falls in the range $20-60 \AA$. Fortunately, the molecular dimensions of most of the proteins also fall within the same range. Besides this, cell membrane thickness and the distance between different subunits of proteins are also of the same order. Consequently, FRET has been used to measure in vivo protein-protein interactions, protein folding kinetics ${ }^{6}$ and protein subunit exchange. Recently, Schuler et $a l^{7}$ using single molecule fluorescence spectroscopy probed the free energy surface for protein folding giving information about the height of free-energy barriers which the conventional equilibrium and kinetic studies fail to yield. The basic concepts of FRET experiments employed by them have been illustrated in figures $3 \mathrm{a}$ and $\mathrm{b}$. They labeled the termini of protein CspTm with green fluorescent donor dye and a red fluorescent acceptor. The concept behind the experiment was that if a folded CspTm molecule diffuses into the volume illuminated by a focused laser beam, then there is rapid EET from D to A as termini are separated only by $10 \AA$ with most of the fluorescence photons emitted by the acceptor. Upon addition of chemical denaturant, the protein unfolds and consequently EET decreases which lower the fraction of photons emitted by the acceptor. Using these single-molecule FRET measurements Schuler et al ${ }^{7}$ were able to calculate limits on the polypeptide reconfiguration time and, according to Kramers theory, the free-energy barrier height can be obtained once the polypeptide reconfiguration time is known from the following equation.

$$
\tau_{f}=\frac{2 \pi \omega_{\min } \tau_{0}}{\omega_{\max }} \exp \left(\frac{\Delta}{k_{B} T}\right),
$$

where $\tau_{f}$ is the folding time, $\omega_{\min }$ and $\omega_{\max }$ are respectively, the frequencies characterizing the curvature of the free energy surface of harmonic well of the unfolded state and at the barrier top, $\Delta$ is the height of the folding free energy barrier and $\tau_{0}$ is the reconfiguration time in unfolded state. Finally, they could obtain the upper and lower bounds, $11 k_{B} T>$ $\Delta>4 k_{B} T$, on the free energy barrier to folding, which otherwise are difficult to obtain.

The other applications include: (a) Probing the biological membrane organization and dynamics ${ }^{8,9}$ as FRET is highly sensitive to distances and changes in the environment of the fluorophore, (b) in medical tests like enzyme-linked immunoassays (ELISA) and fluorescence polarization immunoassays because handling radioactive substances is risky, (c) examining primary and secondary structure of DNA, ${ }^{10}$ translocation of genes between two chromophores, formation of hairpin structures, DNA interaction with drugs, DNA-protein interaction ${ }^{11}$ and in automated DNA sequencing.

\section{FRET in polymers}

In polymer science, FRET is used to study the interface thickness in polymer blends, phase separation and conformational dynamics of polymers. The technique of FRET is being exploited to design supramolecular systems that can be employed to harvest light in artificial photosynthesis as these light-harvesting systems of plants and bacteria involve unidirectional transfer of absorbed radiation energy to the reaction centre via a multistep FRET mechanism. Besides these, FRET is commonly used in scintillators and chemical sensors.

The rest of this section elaborates a few of the points mentioned above and discusses the implica- 

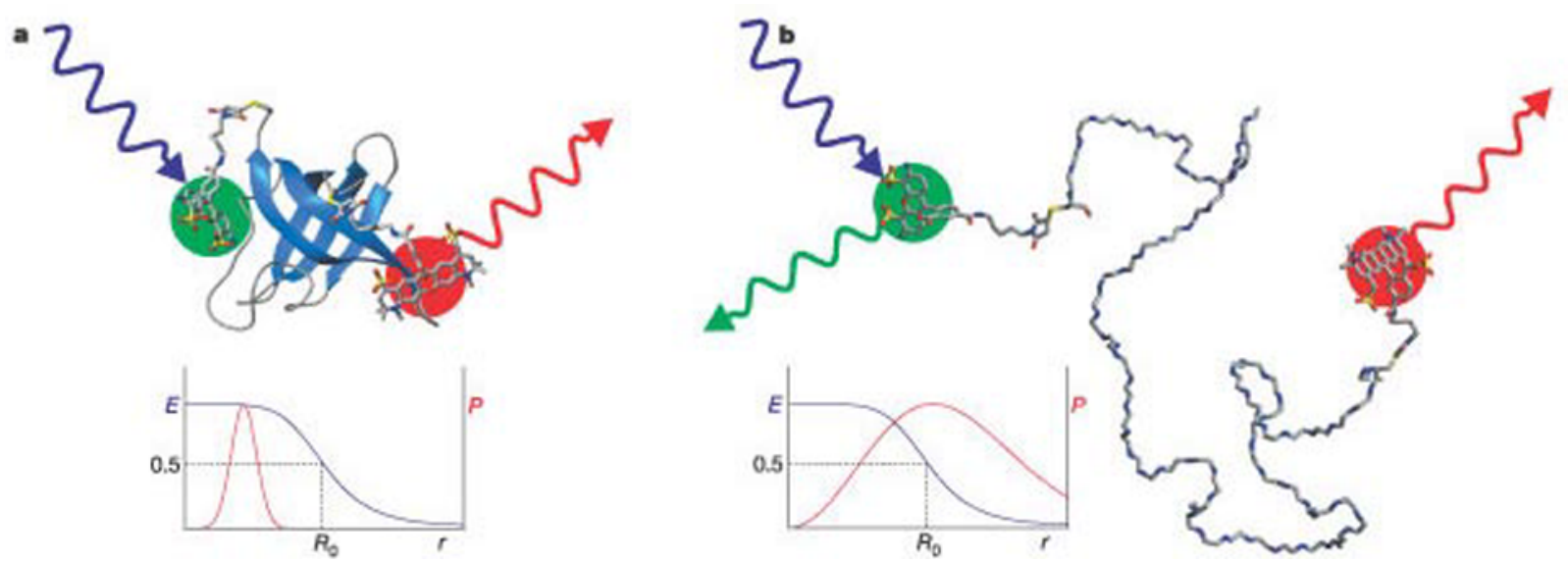

Figure 3. Schematic structures of protein helix labeled with donor (green) and acceptor (red). (a) Folded Csp Tm. (b) Unfolded Csp Tm. In each case, functional form of FRET efficiency $E$ versus distance is shown alongwith representation of the probability distribution of distances between donor and acceptor dyes. (Reprinted with permission from Macmillan Publisher Ltd: Nature (ref. [7]), copyright (2002).)

tions of various other studies undertaken in the past few years.

\subsection{Polymer dynamics}

FRET applied at the single-molecule level is a powerful means for observing the dynamic structural changes in polymers as well as in subpopulations in heterogeneous mixtures. ${ }^{6}$ Time-domain measurements of the decay of the fluorescence intensity from donors are commonly employed to investigate the dynamics of Förster energy migration. Since $k_{\mathrm{rad}}$ and $R_{F}$ both are determined by D-A separation which for flexible molecules is a fluctuating quantity, the rate of decay of fluorescence intensity provides a direct probe for monitoring the conformational dynamics of the polymer.

In order to underline the reliability of FRET as a dynamic marker in the polymer-folding process, Brownian dynamics simulations on model homopolymer systems were carried out by Srinivas and Bagchi. ${ }^{12}$ In these studies, survival probability, $S_{p}(t)$ defined as the probability of reaction between the D-A pair (reaction implies transfer of energy between donor and acceptor) was used to study the folding dynamics. As clear from the definition, $S_{p}(t)$ is a theoretical counterpart of the fluorescence intensity which by itself is a measure of 'un-reacted' donor concentration. For FRET to be useful in the study of folding, it is essential: (1) To choose a value of $R_{F}$ that allow study of both folded and unfolded states as FRET, in principle, is capable of providing information about structural changes only for certain values of $R_{F}$; (2) the three time scales $\tau_{\mathrm{E}, \text { fold }}, \tau_{\mathrm{E} \text {,unfold }}$ and $\tau_{\text {, fold }}$ to obey $\tau_{\mathrm{E} \text {,fold }} \ll \tau_{\text {q,fold }} \ll \tau_{\mathrm{E} \text {,unfold }}$ condition where $\tau_{\mathrm{E}, \text { fold }}$ and $\tau_{\mathrm{E} \text {,unfold }}$ are time scales corresponding to the average $S_{p}(t)$ of FRET in equilibrium folded and unfolded states respectively, while $\tau_{\mathrm{q} \text {,fold }}$ is the time required for polymer to fold subsequent to a quench in the temperature.

Figure 4 shows the decay of $S_{p}(t)$ as obtained from $\mathrm{BD}$ simulations during the folding process. It can be seen that during the folding process, $S_{p}(t)$ follows the decay path of the unfolded state for $t<100 \tau$ after which it starts deviating and decay becomes more rapid. Important to note that the major part of FRET occurs within a time of $200 \tau$, which is same as the average time taken by the polymer to collapse approximately to half of the mean square end-to-end distance of its initial unfolded configuration (compare figures 4 and 5). Also it was observed that the time taken for the D-A pair to react is almost three orders of magnitude slower than that for the folded state. In a separate study, ${ }^{13}$ FRET efficiency distributions were used to obtain important structural parameters like size of the globule, length of the stiff rod etc.

Recently, FRET studies of a series of oligodeoxythymidylates, $(\mathrm{d} T)_{n}$ over a wide range of salt concentration and chain lengths has been performed to test the validity of various statistical approaches, such as flexibly jointed or worm-like chain models often 
used to describe the flexibility and conformations of ssDNA in solution. ${ }^{14}$

Undoubtedly, FRET is very useful technique but it has several limitations as discussed below and in sections to follow.

\subsection{Conjugated polymers}

As already discussed, EET is a basic function of photosynthetic antennas which collect and channel

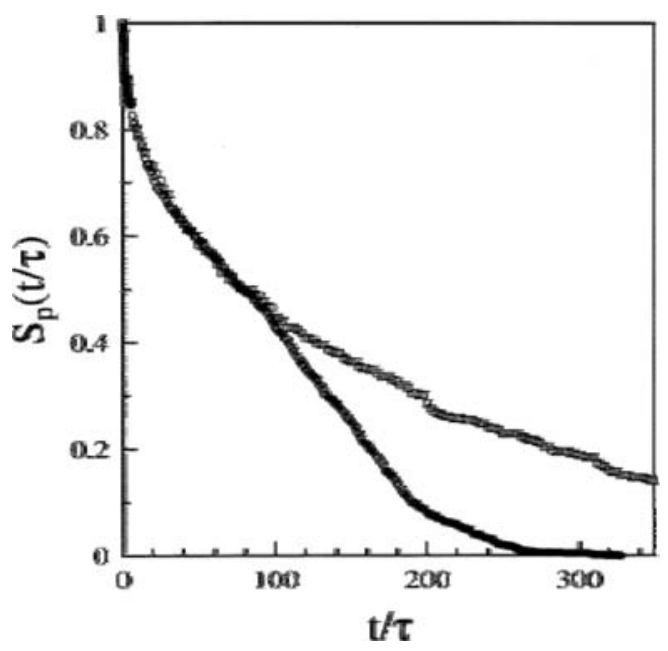

Figure 4. Survival probability for the polymer during the folding process (lower curve) compared to the same function for the equilibrium unfolded state (upper curve).

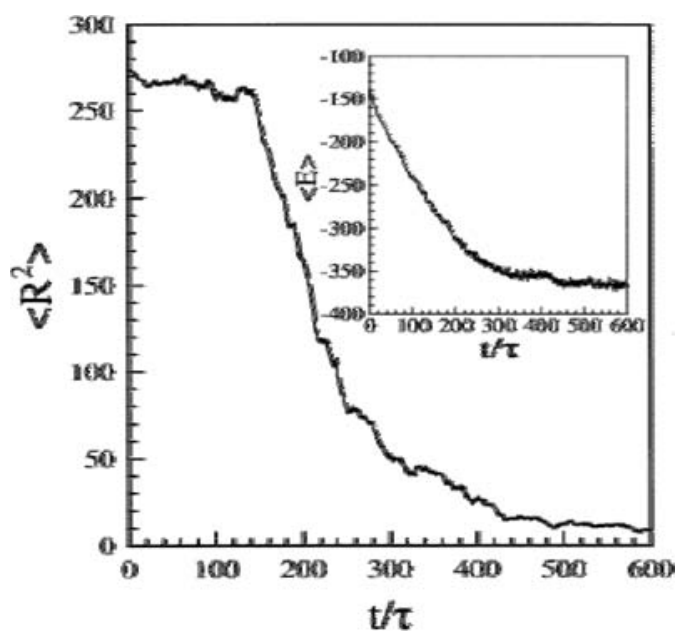

Figure 5. The mean square end-to-end distance $\left\langle R^{2}\right\rangle$ and the average total energy (inset) as a function of time during folding process. the harvested solar energy to the reaction centre with about $95 \%$ efficiency. ${ }^{15}$ These efficient light-harvesting systems are nothing but extensively conjugated organic systems. As a consequence, the process of EET in conjugated systems is being foreseen as a mode of signal transmission in molecular electronics. Moreover, these systems are already finding a number of applications in display devices. ${ }^{16,17}$

To design systems as efficient as photosynthetic antennas, the first step is to direct the flow of energy towards the desired regions. To attain this objective, scientists have linked chromophores with continually decreasing band gaps along the polymer chains, ${ }^{18}$ for the reasons already discussed. However, in such systems energy is lost because of large differences required in the emission spectra of successive chromophores.

A novel system that controls the energy flow more efficiently has been designed by Schwartz et $a l^{19}$ It involve the chains of semiconducting poly[2methoxy-5-(2'-ethyl-hexyloxy)-1,4-phenylene vinylene] (MEH-PPV) aligned and encapsulated into the hexagonally arrayed channels of mesoporous silica glass. PPV and hence its water soluble derivative, MEH-PPV, are not infinitely long conjugated systems but consist of chains of conjugated phenylvinyl oligomers of various lengths because of bends and twists in the polymer chain. These oligomers can serve both as acceptor and donor molecules in non-radiative excitation transfer as excitation energy is a strong function of conjugation length.

In this system, the polymer outside the channel is composed of short randomly oriented oligomers having high excitation energy, while the portion inside oriented along the channel consists of longer segments. This particular design directs the energy deposited with the randomly oriented segments towards the aligned ones inside the channels. With the help of both steady-state and time-resolved luminescence measurements, Schwartz et al concluded that the interchain energy transfer (energy transfer between the randomly oriented chains outside the channels) is the Förster transfer.

Since the interchain migration rate depends on the relative internal geometries of the donor and acceptor chromophores, therefore, an understanding of spatial and orientation dependence of the rate of excitation energy transfer is important for optimizing the performance of molecular-based devices involved in EET. Wong et $a l^{20}$ have investigated this dependence for a six-unit oligomer of polyfluorene $\left(\mathrm{PF}_{6}\right)$ and tetraphenylporphyrin (TPP), which brought for- 


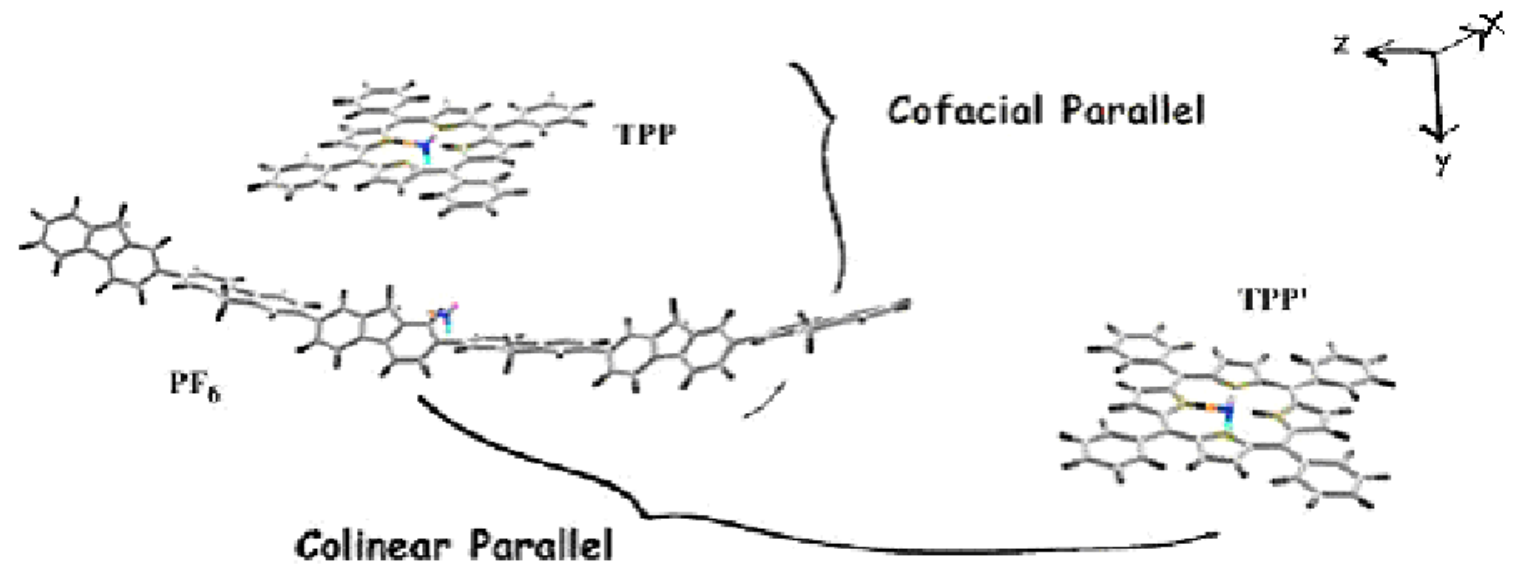

Figure 6. Schematic representation of donor chromophore $\mathrm{PF}_{6}$ and the acceptor TPP in an arrangement where the transition dipole moments are aligned (1) parallel to each other and (2) orthogonal to DA intermolecular axis (cofacial parallel) and parallel to the DA intermolecular axis (colinear parallel). $z$-axis shows the direction of the transition dipole moment vector.

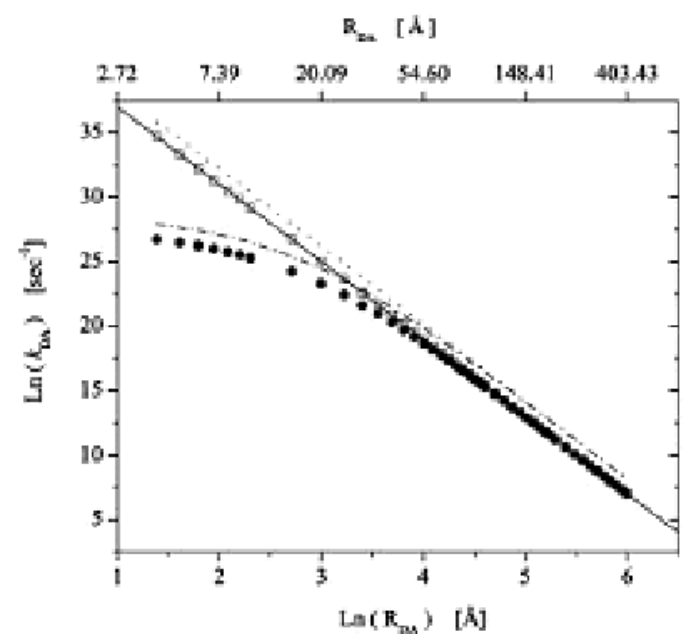

Figure 7. Distance dependence of the rate for the cofacial parallel orientation of donor and acceptor $((\square)$ Förster rate and $(\bullet)$ resonance-Coulomb rates) for EET between the donor state and the acceptor state having mid-range oscillator strength. The traditional $R^{-6}$ distance dependence is shown by the solid line, the total Förster and resonance-Coulomb rates, summed over states are represented by dotted and dashed lines, respectively.

ward several limitations of the Förster theory. The computational approach employed semiempirical Pariser-Parr-Pople (PPP) hamiltonian coupled with single configuration interaction (SCI). From the PPP/SCI wave functions, electronic transition energies, and transition dipole moments, the full resonanceCoulomb coupling matrix elements as well as the point-dipole approximation to the coupling was computed.

The comparison of the calculated distance and orientation dependence of Förster rate to the full resonance-Coulomb rate from identical wave functions clearly delineated the limitations of the point-dipole formulation, which is invalid at short D-A separations. The plot of the rate dependence of EET between the donor state and the acceptor state having mid-range oscillator strength $(0.70)$ against the DA separation (figure 7) shows that the transitions to these acceptor states dominate the total rate. The separate calculation of transfer rate between optically dark states of D and A shows the rate to be of the same order as that between the optically bright states suggesting that though these optically dark states do not contribute to absorption spectrum of the acceptor but can mediate EET. Moreover, the plot clearly indicates the violation of Förster distance-dependence at small DA separations. This difference from the Förster's macroscopic formulation is a manifestation of the breakdown of the point-dipole approximation.

Figure 8 shows the orientation dependence for the cofacial case (figure 6) for two DA separation distances, 10 and $100 \AA$. Here, the angle $\theta$ corresponds to the rotation of TPP acceptor molecule about the transition dipole moment axis ( $z$-axis in figure 6 ). Figure $8 \mathrm{a}$ shows that the rate varies by a factor of $\sim 2$ in going from $0^{\circ}$ to $90^{\circ}$, whereas the dipole approximation to the rate shows negligible dependence. However, at large separations (figure 8b) the EET rate shows weak orientation dependence. 

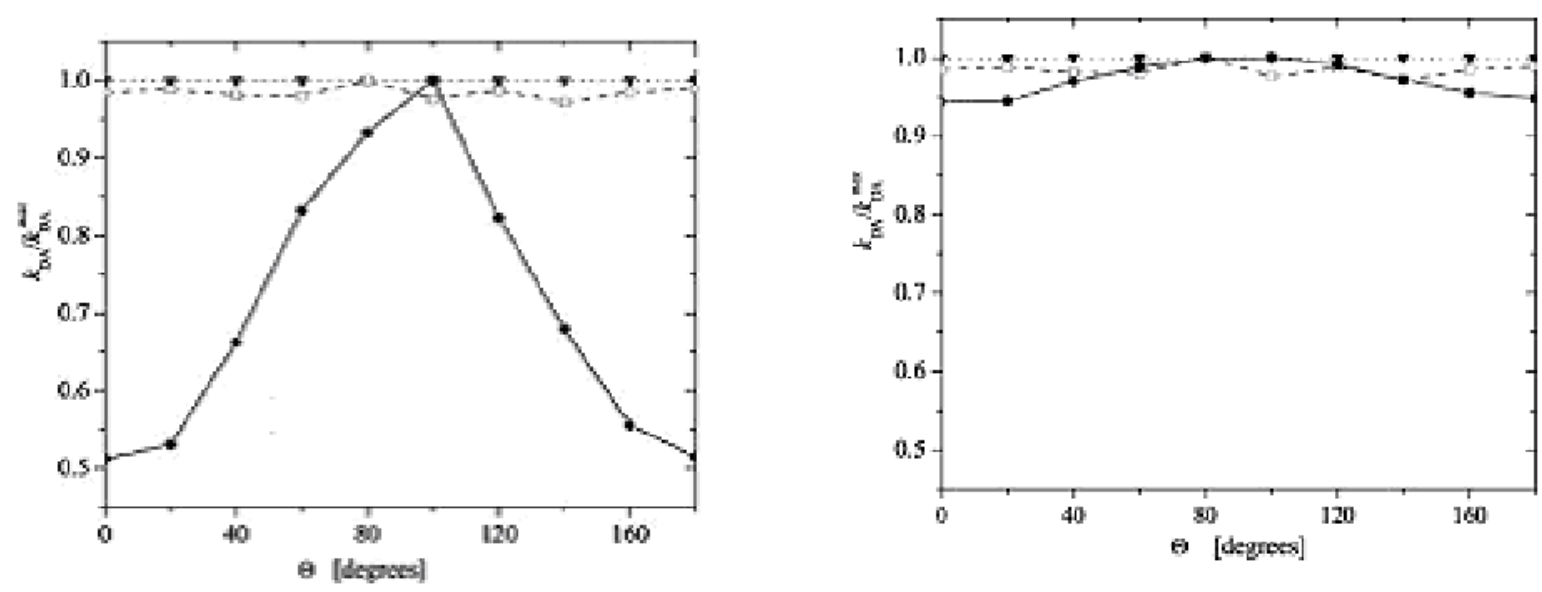

Figure 8. Orientation dependence of normalized rate at (a) short $(10 \AA)$ and (b) long (100 $\mathrm{A})$ DA separation for an initial cofacial parallel alignment of DA transition moments $((\square)$ Förster rate and $(\bullet)$ resonance-Coulomb rates).

In brief, the study by Wong et al proves the inadequacy of the Förster theory at short distances, particularly for extended conjugated systems where the transition dipole densities are distributed on the length scale similar to DA separation.

\section{FRET among nanoparticles}

FRET technology is very useful and can be applied at single-molecule levels to observe dynamic changes but the length-scale over which these measurements can be made is limited by the nature of dipole-dipole mechanism. Therefore, to study large, multi-component complexes like ribosomes or various nucleoprotein complexes, there is need to develop new optical methods which can investigate both longrange static and dynamic distances with least interference in biomolecular functions.

As has been pointed out by Yun et al, ${ }^{21}$ this limitation of FRET can be overcome by introducing additional transition dipoles, which provide more coupling interactions. The physical consequences of these interactions can be understood from the Fermigolden rule approach that relates the energy transfer $\left(k_{\text {Ent }}\right)$ to a product of interaction elements of donor $\left(F_{D}\right)$ and the acceptor $\left(F_{A}\right)$ as $k_{\text {Ent }} \approx F_{D} F_{A}$. In much simplified form, assuming these interactions to be the sole function of their DA separation, one obtains $F \approx 1 / R^{3}$ for single dipoles, $F \approx 1 / R$ for $2 \mathrm{D}$ dipole array and $F \approx$ constant for $3 \mathrm{D}$ dipole array. The rate constant for FRET which consists of two single dipoles can be easily derived from this approach, $k_{F} \approx$
$F_{D} F_{A} \approx\left(1 / R^{3}\right)\left(1 / R^{3}\right) \approx 1 / d^{6}$, while the rate constant for energy transfer between metal surface and a dipole follows $1 / d^{4}$ dependence, as $k_{\mathrm{SET}} \approx F_{D} F_{A} \approx\left(1 / R^{3}\right)$ $(1 / R) \approx 1 / R^{4}{ }^{21}$ The $1 / R^{4}$ dependence has been theoretically derived by Chance, Prock and Silbey ${ }^{22}$ by considering the rate of energy transfer from a dipole to a metallic surface. This approach has further been extended by Persson and Lang ${ }^{23}$ to the metal's conduction electrons. The comparison of the experimental energy transfer efficiency values for a system consisting of fluorescein moiety appended to one end of dsDNA and an Au-nanoparticle at the other end, with the theoretical energy transfer curves for a pure dipole-dipole (FRET) and dipole-surface (SET) energy transfer process shows precisely the $\left(1 / R^{4}\right)$ dependence (figure 9) as predicted for a dipole interacting with the metal surface. This suggests that Au-nanoparticle $(1.4 \mathrm{~nm})$ acts like a metal surface with respect to the FAM dipole. ${ }^{21}$ The process of SET originates because of the interaction of electromagnetic field of the donor dipole with the free conduction electrons of the accepting metal. As a result, the dipole does not interact with a discrete resonance electronic transition as in case of FRET but with a continuum of electronic levels of a metallic system providing greater degree of coupling. The distance derivatives of SET and FRET curves of figure 9 plotted in figure 10 show FRET to be a highly sensitive method but over a very narrow-range of distances $(<100 \AA)$, whereas SET though not as efficient as FRET provides good distance resolution even up to $220 \AA$. Therefore, as quoted by Yun et $a l^{21}$, we can imagine FRET as a very short ruler (scale) with 
finely spaced markings while SET is a very long ruler with widely spaced markings.

In a separate study ${ }^{24}$ it has been found that systems with dye-molecules linked directly to nanoparticles have more fluorescence quenching efficiency caused both by increase in non-radiative transfer rate and a drastic decrease in dye's radiative rate. Both the radiative and the non-radiative rates are found to depend critically on the size and shape of the nanoparticle.

The sensitivity and the range of distance measurements, can be increased by the use of systems where both donor and acceptor are nanoparticles. However, the use of these systems in biological de-

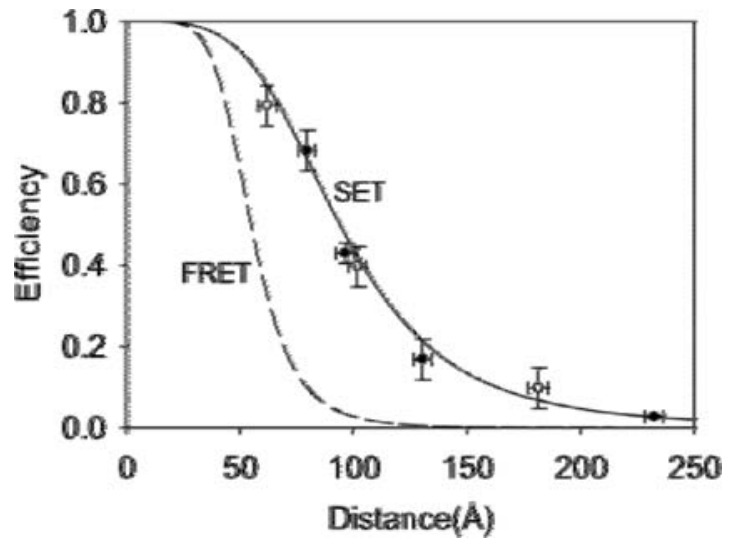

Figure 9. Energy transfer efficiency plotted against separation distance between FAM and $\mathrm{Au}(\mathrm{NM})$. The measured efficiencies of DNA strands of various lengths are represented by open circles $(\mathrm{O})$. The dashed line is the theoretical FRET efficiency, while the solid line is the theoretical SET efficiency. (Reproduced with permission from ref. [21].)

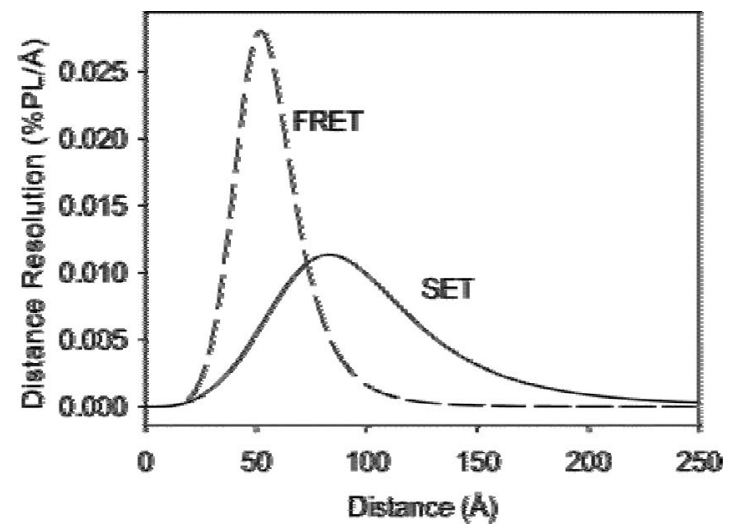

Figure 10. Separation distance-dependent resolution of the FRET and SET mechanisms. (Reproduced with permission from ref. [21].) tection is limited due to their inherent toxicity, chemical instability and high background noise. However, a novel biosensor based on energy transfer between bioconjugated UC (upconversion) nanophosphores and $\mathrm{Au}$ nanoparticles recently has been developed which overcomes these limitations to some extent. ${ }^{25}$ These UC nanoparticles can be excited in the IR region whereas the interfering biomolecules absorb in the UV region causing decrease in the background noise. It is hoped that these upconversion phosphors will find wider application in fluorescence immunoassays and fluorescence imaging, performed both in vivo and in vitro.

Though the study of energy transfer between Aunanoparticles and dyes agrees with the SET expression obtained for metal surfaces, the energy transfer between a donor dye and an acceptor nanoparticle has features which are distinct from that of transfer between a dye and a metal surface. It is also quite distinct from that between two chromophores. The unique features of FRET between a dye and a nanometal particle require separate treatment. Here we outline a simple theory developed recently. ${ }^{26}$

The rate of energy transfer between the acceptor (nanoparticle) and the donor dye molecule can be obtained following the same approach as is adopted for conventional FRET systems in $\$ 2$. In the present case, the coupling matrix elements of (3) are evaluated assuming that the donor dye molecule interacts with the charge distribution of the nanoparticle via a point dipole located suitably, for example, at the molecule's centre of gravity. Hence, we can write $V_{D A}$ as,

$$
V_{\mathrm{DA}}=\int_{V} \mathrm{~d} \mathbf{r}^{\prime} \frac{\hat{\boldsymbol{\mu}}_{\mathrm{D}} \cdot\left(\hat{\mathbf{R}}-\hat{\mathbf{r}}^{\prime}\right)}{\left|\left(\mathbf{R}-\mathbf{r}^{\prime}\right)\right|^{2}} \rho\left(\mathbf{r}^{\prime}\right),
$$

where $V$ is the volume over which charge is distributed, $\hat{\boldsymbol{\mu}}_{\mathrm{D}}$ is the dipole operator for the donor, $\rho\left(\mathbf{r}^{\prime}\right)$ is the charge density distribution, $\mathbf{r}^{\prime}$ being a coordinate with respect to the centre of mass of the charge distribution, $\hat{\mathbf{R}}$ and $\hat{\mathbf{r}}^{\prime}$ are the unit vectors in the direction of $\mathbf{R}$ (distance of separation between interacting systems) and $\mathbf{r}^{\prime}$ respectively as shown in figure 11. Expanding $\left|\left(\mathbf{R}-\mathbf{r}^{\prime}\right)\right|^{2}$ in terms of multipole expansion and retaining only first-order terms, the above expression reduces to

$$
V_{D A}=\hat{\mathbf{\mu}}_{\mathrm{D}} \cdot \int_{V} \mathrm{~d} \mathbf{r}^{\prime}\left(\hat{\mathbf{R}}-\hat{\mathbf{r}}^{\prime}\right)\left[\frac{1}{r \succ}+\frac{\mathbf{r}_{\succ} \hat{\mathbf{r}}_{\succ}}{r_{\succ}^{2}}+Q\right]^{2} \rho\left(\mathbf{r}^{\prime}\right),
$$




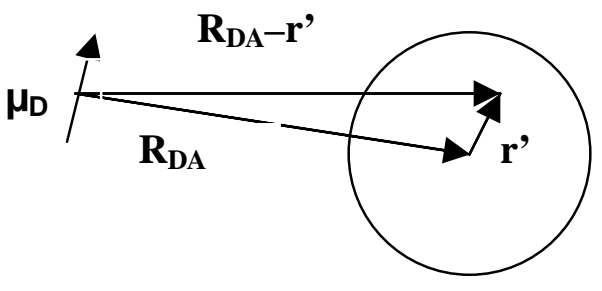

Figure 11. Depicts the arrangement of a dye's dipole with respect to the nanoparticle.

where $\mathbf{r}_{\succ(\curlywedge)}$ is a vector with magnitude $\mathbf{r}_{\succ(\curlywedge)}$ equal to greater (lesser) of $R=|\mathbf{R}|$ and $r^{\prime}=\left|\mathbf{r}^{\prime}\right|$, and $Q$ refers to higher order terms. The above equation can be decomposed into a sum of two terms as given below.

$V_{D A}=\hat{\mu}_{\mathrm{D}} \cdot\left[\begin{array}{c}\int_{r^{\prime} \prec R} \mathrm{~d} \mathbf{r}^{\prime}\left(\hat{\mathbf{R}}-\hat{\mathbf{r}}^{\prime}\right)\left(\frac{1}{R}+\frac{\mathbf{r}^{\prime} \hat{\mathbf{R}}}{R^{2}}+Q\right)^{2} \rho\left(\mathbf{r}^{\prime}\right)+ \\ \int_{r^{\prime} \succ R} \mathrm{~d} \mathbf{r}^{\prime}\left(\hat{\mathbf{R}}-\hat{\mathbf{r}}^{\prime}\right)\left(\frac{1}{r^{\prime}}+\frac{\mathbf{R} \cdot \hat{\mathbf{r}}^{\prime}}{r^{2}}+Q\right)^{2} \rho\left(\mathbf{r}^{\prime}\right)\end{array}\right]$.

Using (14), $J_{D A}$ in the present case represents the electronic contribution to coupling and is given by,

$$
\begin{aligned}
& J_{D A}=\left\langle\phi_{e}^{D}, \phi_{g}^{A}\right| \hat{\mu}_{\mathrm{D}} . \\
& {\left[\begin{array}{l}
\left.\int_{r^{\prime} \prec R} \mathrm{~d} \mathbf{r}^{\prime}\left(\hat{\mathbf{R}}-\hat{\mathbf{r}}^{\prime}\right)\left(\frac{1}{R}+\frac{\mathbf{r}^{\prime} \cdot \hat{\mathbf{R}}}{R^{2}}+Q\right)^{2} \rho\left(\mathbf{r}^{\prime}\right)+\right] \\
\int_{r^{\prime} \succ R} \mathrm{~d} \mathbf{r}^{\prime}\left(\hat{\mathbf{R}}-\hat{\mathbf{r}}^{\prime}\right)\left(\frac{1}{r^{\prime}}+\frac{\mathbf{R} \cdot \hat{\mathbf{r}}^{\prime}}{r^{2}}+Q\right)^{2} \rho\left(\mathbf{r}^{\prime}\right)
\end{array}\right]\left|\phi_{e}^{A}, \phi_{g}^{D}\right\rangle .}
\end{aligned}
$$

It can be rewritten as,

$$
\begin{aligned}
& J_{D A}=\mu_{D} \cdot\left\langle\phi_{g}^{A}\right|
\end{aligned}
$$

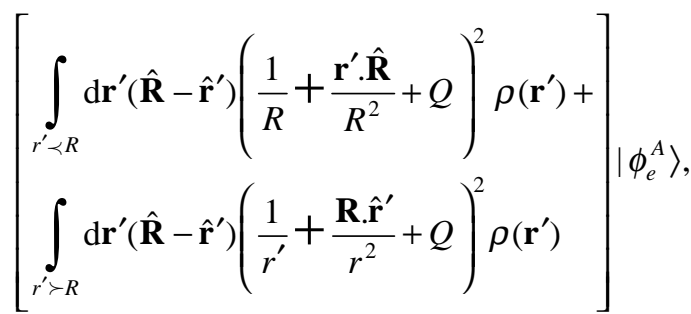

where $\mu_{D}=\left\langle\phi_{e}^{D}\left|\hat{\mu}_{D}\right| \phi_{g}^{D}\right\rangle$.

Assuming that charge density may be written as the sum over contributions from individual electronic charges, we have

$$
\int \mathrm{d} \mathbf{r}^{\prime} f\left(\mathbf{r}^{\prime}\right) \rho\left(\mathbf{r}^{\prime}\right)=\sum_{i} \rho_{i}\left(\mathbf{r}^{\prime}\right) f\left(\mathbf{r}^{\prime}\right),
$$

where $\rho_{i}\left(\mathbf{r}^{\prime}\right)$ is the contribution to the total charge density from the $i$ th electron in the nanoparticle and $f\left(\mathbf{r}^{\prime}\right)$ is some continuous function of $\mathbf{r}^{\prime}$. Substituting it in (16), $J_{D A}$ becomes

$$
\begin{aligned}
& J_{D A}=\mu_{\mathrm{D}} \cdot \sum_{i} \\
& {\left[\begin{array}{c}
\left\langle\phi_{g}^{A}\left|\left(\hat{\mathbf{R}}-\hat{\mathbf{r}}^{\prime}\right)\left(\frac{1}{R}+\frac{\mathbf{r}^{\prime} \cdot \hat{\mathbf{R}}}{R^{2}}+Q\right)^{2} \rho_{i}\left(\mathbf{r}^{\prime}\right)\right| \phi_{e}^{A}\right\rangle_{r^{\prime}<R} \\
+\left\langle\phi_{g}^{A}\left|\left(\frac{1}{r^{\prime}}+\frac{\mathbf{R} \cdot \hat{\mathbf{r}}^{\prime}}{r^{\prime 2}}+Q\right)^{2} \rho_{i}\left(\mathbf{r}^{\prime}\right)\right| \phi_{e}^{A}\right\rangle_{r^{\prime} \succ R}
\end{array}\right] .}
\end{aligned}
$$

Finally, retaining only first-order terms, (17) simplifies to give

$$
J_{D A}=\boldsymbol{\mu}_{\mathrm{D}} \cdot \sum_{i}\left[\begin{array}{l}
\frac{1}{R^{2}}\left\langle\phi_{g}^{A}\left|\left(\hat{\mathbf{R}}-\hat{\mathbf{r}}^{\prime}\right) \rho_{i}\left(\mathbf{r}^{\prime}\right)\right| \phi_{e}^{A}\right\rangle_{r^{\prime} \prec R} \\
+\left\langle\phi_{g}^{A}\left|\left(\hat{\mathbf{R}}-\hat{\mathbf{r}}^{\prime}\right) \frac{\rho_{i}\left(\mathbf{r}^{\prime}\right)}{r^{\prime 2}}\right| \phi_{e}^{A}\right\rangle_{r^{\prime} \succ R}
\end{array}\right] .
$$

Typically, for a metallic nanoparticle $\left\langle\phi_{g}^{A}\right|$ and $\left\langle\phi_{e}^{A}\right|$ are excitonic states undergoing plasmonic excitations. The corresponding wavefunctions are delocalized all over the nanoparticle with the extent of delocalization being dependent on the size of the system.

Now, let us consider two separate cases.

(A) Case 1: When the size of the nanoparticle is comparable or larger than the nanoparticle-dye separation.

For such a system, contribution from the first term decreases because of the additional factor $\left(r^{\prime} / R\right)^{2}$ which has a value in a range $(0,1)$ while that from the second term, which is independent of $R$, increases as the volume of integration has increased. This can easily be seen by writing the radial and polar integration variables separately as is done below. 


$$
\begin{aligned}
J_{D A} & =\boldsymbol{\mu}_{\mathrm{D}} \cdot \sum_{i} \\
\int \mathrm{d} \Omega^{\prime} & {\left[\begin{array}{c}
\frac{1}{R^{2}} \int_{r^{\prime}} \mathrm{d} r^{\prime} r^{\prime 2} \phi_{g}^{A^{*}}\left(r^{\prime}\right)\left(\hat{\mathbf{R}}-\hat{\mathbf{r}}^{\prime}\right) \rho_{i}\left(\mathbf{r}^{\prime}\right) \phi_{e}^{A}\left(r^{\prime}\right) \\
\quad+\int_{r^{\prime} \succ R} \mathrm{~d} r^{\prime} \phi_{g}^{A^{*}}\left(r^{\prime}\right)\left(\hat{\mathbf{R}}-\hat{\mathbf{r}}^{\prime}\right) \rho_{i}\left(\mathbf{r}^{\prime}\right) \phi_{e}^{A}\left(r^{\prime}\right)
\end{array}\right] . }
\end{aligned}
$$

As a result, the variation of $J_{D A}$ with $R$ will be quite complex and weaker than $R^{-2}$.

In general terms, the absolute square of $J_{D A}$ can be written as,

$$
\left|J_{D A}\right|^{2}=\left|\mu_{D}\right|^{2}\left(a_{0}+\frac{a_{2}}{R^{2}}+\frac{a_{4}}{R^{4}}\right) P_{\text {orient. }}^{2},
$$

where $P_{\text {orient }}$ takes into account the orientation factor.

Thus (3) becomes,

$$
\begin{aligned}
& \left|\left\langle\psi_{e M_{D}}^{D}, \psi_{g N_{A}}^{A}\left|V_{D A}\right| \psi_{e M_{A}}^{A}, \psi_{g N_{D}}^{D}\right)\right|^{2} \\
& \quad=\left.\left|\boldsymbol{\mu}_{D}\right|^{2}\left(a_{0}+\frac{a_{2}}{R^{2}}+\frac{a_{4}}{R^{4}}\right)\left\langle\chi_{D e} \mid \chi_{D g}\right\rangle\left\langle\chi_{A g} \mid \chi_{A e}\right\rangle\right|^{2} P_{\text {orient }}^{2},
\end{aligned}
$$

where

$$
\begin{aligned}
a_{0}= & \sum_{i}\left|\left\langle\phi_{g}^{A}\left|\left(\hat{\mathbf{R}}-\hat{\mathbf{r}}^{\prime}\right) \frac{\rho_{i}\left(r^{\prime}\right)}{r^{\prime 2}}\right| \phi_{e}^{A}\right\rangle_{r^{\prime} \succ R}\right|^{2}, \\
a_{2}= & 2 \sum_{i}\left|\left\langle\phi_{g}^{A}\left|\left(\hat{\mathbf{R}}-\hat{\mathbf{r}}^{\prime}\right) \frac{\rho_{i}\left(r^{\prime}\right)}{r^{\prime 2}}\right| \phi_{e}^{A}\right\rangle_{r^{\prime} \succ R}\right|, \text { and } \\
& \left|\left\langle\phi_{g}^{A}\left|\left(\hat{\mathbf{R}}-\hat{\mathbf{r}}^{\prime}\right) \rho_{i}\left(\mathbf{r}^{\prime}\right)\right| \phi_{e}^{A}\right\rangle_{r^{\prime} \prec R}\right| \\
a_{4}= & \sum_{i}\left|\left\langle\phi_{g}^{A}\left|\left(\hat{\mathbf{R}}-\hat{\mathbf{r}}^{\prime}\right) \rho_{i}\left(\mathbf{r}^{\prime}\right)\right| \phi_{e}^{A}\right\rangle_{r^{\prime} \prec R}\right|^{2} .
\end{aligned}
$$

Here the contribution from $a_{0}$ term will be the maximum.

Using (2), (5) and (21), the rate expression becomes,

$$
\begin{aligned}
& k_{\text {non-rad }}=\frac{2 \pi P_{\text {orient }}^{2}}{\hbar} \sum_{D} \sum_{A} f\left(E_{D e}\right) f\left(E_{A g}\right)\left|\boldsymbol{\mu}_{D}\right|^{2} \\
& \left(a_{0}+\frac{a_{2}}{R^{2}}+\frac{a_{4}}{R^{4}}\right)\left|\left\langle\chi_{D e} \mid \chi_{D g}\right\rangle\left\langle\chi_{A g} \mid \chi_{A e}\right\rangle\right|^{2} \\
& \times \int_{-\infty}^{+\infty} \delta\left(E_{D e}-E_{D g}-E\right) \times \delta\left(E+E_{A g}-E_{A e}\right) \mathrm{d} E .
\end{aligned}
$$

Since the $a_{0}$ term contributes the maximum to $\left|J_{D A}\right|^{2}$, for such systems the distance dependence of the rate of energy transfer is weaker than $R^{-4}$.

(B) Case 2: When the size of the nanoparticle is smaller than the nanoparticle-dye separation.

In such a case with $r^{\prime}<R,(18)$ reduces to

$$
J_{D A}=\boldsymbol{\mu}_{D} \cdot \sum_{i}\left[\frac{1}{R^{2}}\left\langle\phi_{g}^{A}\left|\left(\hat{\mathbf{R}}-\hat{\mathbf{r}}^{\prime}\right) \rho_{i}\left(\mathbf{r}^{\prime}\right)\right| \phi_{e}^{A}\right\rangle\right]
$$

with

$$
\begin{aligned}
& \left|J_{D A}\right|^{2}=\frac{\left|\boldsymbol{\mu}_{D}\right|^{2}}{R^{4}} \\
& \left|\sum_{i}\left[\left\langle\phi_{g}^{A}\left|\left(\hat{\mathbf{R}}-\hat{\mathbf{r}}^{\prime}\right) \rho_{i}\left(\mathbf{r}^{\prime}\right)\right| \phi_{e}^{A}\right\rangle\right]\right|^{2} P_{\text {orient. }}^{2}
\end{aligned}
$$

Using (2), (5) and (21), the rate expression becomes,

$$
\begin{aligned}
k_{\text {non-rad }}= & \frac{2 \pi P_{\text {orient }}^{2}}{\hbar} \frac{1}{R^{4}} \sum_{D} \sum_{A} f\left(E_{D e}\right) f\left(E_{A g}\right)\left|\boldsymbol{\mu}_{D}\right|^{2} \\
& \left|\sum_{i}\left[\left\langle\phi_{g}^{A}\left|\left(\hat{\mathbf{R}}-\hat{\mathbf{r}}^{\prime}\right) \rho_{i}\left(\mathbf{r}^{\prime}\right)\right| \phi_{e}^{A}\right\rangle\right]\right|^{2} \\
\times & \left|\left\langle\chi_{D e} \mid \chi_{D g}\right\rangle\left\langle\chi_{A g} \mid \chi_{A e}\right\rangle\right|^{2} \\
& \times \int_{-\infty}^{+\infty} \delta\left(E_{D e}-E_{D g}-E\right) \times \delta\left(E+E_{A g}-E_{A e}\right) \mathrm{d} E .
\end{aligned}
$$

For such systems we find the expected $R^{-4}$ distance dependence of the rate of energy transfer.

Now, all we need is to find out a spectroscopic means similar to (6), so that for these systems as well we can express the coupling as a spectral overlap between the donor fluorescence and the acceptor absorbance. As in $\$ 2$, we can write donor fluorescence, $f_{D}(\omega)$ as,

$$
\begin{aligned}
& f_{D}(\omega)=\text { constant } \times\left|\mu_{D}\right|^{2} \sum_{D} f\left(E_{D e}\right)\left|\left\langle\chi_{D e} \mid \chi_{D g}\right\rangle\right|^{2} \\
& \times \delta\left(E_{D e}-E_{D g}-\hbar \omega\right)
\end{aligned}
$$

while for acceptor absorbance, let us consider case 2 , 


$$
\begin{aligned}
& a_{A}(\omega)=C_{A} \sum_{A} f\left(E_{A g}\right) \\
& \quad\left|\sum_{i}\left[\left\langle\phi_{g}^{A}\left|\left(\hat{\mathbf{R}}-\hat{\mathbf{r}}^{\prime}\right) \rho_{i}\left(\mathbf{r}^{\prime}\right)\right| \phi_{e}^{A}\right\rangle\right]\right|^{2}\left|\left\langle\chi_{A g} \mid \chi_{A e}\right\rangle\right|^{2} \\
& \quad \times \delta\left(E_{A g}-E_{A e}+\hbar \omega\right) .
\end{aligned}
$$

Using the above equations, the rate expression (24) can be written as,

$$
k_{\text {non-rad }}=\frac{k_{\text {rad }}}{R^{4}} \int_{0}^{\infty} F\left(\frac{1}{\omega}\right) a_{A}(\omega) f_{D}(\omega) \mathrm{d} \omega
$$

where $F(1 / \omega)$ is a function of $(1 / \omega)$. Equation (25) derived in the present study is similar to (6) except that the exact form of $F(1 / \omega)$ is not known. In the Förster case, $F(1 / \omega)$ is $1 / \omega^{4}$, while in the study reported by Yun et $a l^{21} F(1 / \omega)$ is given as $1 / \omega^{3}$.

This study demonstrates qualitatively much weaker distance-dependence compared to the Förster rate expression when the size of the acceptor becomes comparable to or is larger than the distance of separation between donor and acceptor. However, at very small separation when the orbitals of acceptor and donor start overlapping, the Dexter mechanism ${ }^{27}$ dominates resulting in exponential variation of rate with $R$. However, we believe that there may still be a considerable range of $R$ where Coulombic energy transfer is the dominant mechanism, with nonFörster distance-dependence of the rate of excitation transfer.

\section{Conclusions}

No doubt FRET is a powerful technique and has rightly been referred to as "spectroscopic molecular ruler". Like any other technique it too has several limitations like applicability in a narrow range of distances, uncertainty in the orientation factor and breakdown of the validity of Förster simple expression for extended charge distributions as in the case of conjugated systems and nanoparticles. In these systems, the distance dependence can be found as weak as $R^{-2}$. Note that $R^{-4}$ dependence has already been found in the case of nanoparticles.

Studies have shown that Förster formalism which provide theoretical basis for FRET has certain flaws as, for example, dipole-dipole approximation, weak coupling limit and the assumption of fast vibrational relaxation of the donor state prior to energy transfer.
The influence of the last assumption on Förster rate remains poorly understood. ${ }^{28}$

All the results and arguments presented in this paper show the need to go beyond the accepted Förster approach.

\section{Acknowledgements}

We thank Prof P Rossky and Dr Kim Wong for collaboration in the conjugated polymer problem. We thank Prof D Birch and Prof S Natarajan for useful discussions on FRET. This work was supported in parts by a grant from the Department of Science and Technology, New Delhi. HS thanks the SSCU faculty and staff for hospitality during his visit to IISc, Bangalore.

\section{References}

1. Förster Th 1948 Ann. Phys. (Leipzig) 255

2. Förster Th 1965 in Modern quantum chemistry, istanbul lectures, Part III: Action of light and organic crystals (ed.) O Sinanoglu (New York: Academic Press) pp 93-137

3. Perrin J 1927 C. R. Acad. Sci. (Paris) 1841097

4. Scholes G D 2003 Annu. Rev. Phys. Chem. 5457

5. Lakowicz J R 1983 Principles of fluorescence spectroscopy (New York: Plenum)

6. Deniz A A, Dahan M, Grunwell J R, Ha T, Faulhaber A E, Chemla D S, Weiss S and Schultz P G 1999 Proc. Natl. Acad. Sci. USA 963670

7. Schuler B, Lipman E A and Eaton W A 2002 Nature (London) 419743

8. Struck D K, Hoeskstra D and Pagano R E 1981 Biochemistry 204093

9. Dumas F, Sperotto M M, Lebrun M C, Tocanne T F and Mouritsen O G 1997 Biophys. J. 731940

10. Clegg R M, Murchie A I H, Zechel A and Lilley M J 1993 Proc. Natl. Acad. Sci. USA 902994

11. Issac V E, Patel L, Curran T and Abate-Shen C 1995 Biochemistry 3415276

12. Srinivas G and Bagchi B 2001 J. Phys. Chem. B105 2475

13. Srinivas G and Bagchi B 2002 J. Chem. Phys. 116 837

14. Murphy M C, Rasnik I, Cheng W and Ha T 2004 Biophys. J. 862530

15. Orritt M 1999 Science 285 349, and references therein

16. Tasch S, List E J W, Hochfilzer C, Leising G, Schlichting P, Rohr U, Geerts Y, Scherf U and Mullen K 1997 Phys. Rev. B56 4479

17. Hu B, Zhang N and Karasz F E 1998 J. Appl. Phys. 836002

18. Fox M A 1999 Acc. Chem. Res. 32 201, and references therein 
19. Nguyen T, Wu J, Doan V, Schwartz B J and Tolbert S H 2000 Science 288652

20. Wong K F, Bagchi B and Rossky P J 2004 J. Phys. Chem. A108 5752

21. Yun C S, Javier A, Jennings T, Fisher M, Hira S, Peterson S, Hopkins B, Reich N O and Strouse G F 2005 J. Am. Chem. Soc. 1273115

22. Chance R R, Prock A and Silbey R 1978 Adv. Chem. Phys. 371
23. Persson B and Lang N 1982 Phys. Rev. B26 5409

24. Dulkeith E, Morteani A C, Niedereichholz T and Feldmann J 2002 Phys. Rev. Lett. 89203002

25. Wang L, Yan R, Huo Z, Wang L, Zeng J, Bao J, Wang X, Peng Q and Li Y 2005 Angew. Chem. Int. Ed. 446054

26. Singh H and Bagchi B 2005 Curr. Sci. 891710

27. Dexter D L 1953 J. Chem. Phys. 21836

28. Yang M and Fleming G R 2002 Chem. Phys. 55457 